\title{
Forecasting Using Alternative Measures of Model-Free Option-Implied Volatility
}

\author{
Xingzhi Yao and Marwan Izzeldin \\ September 12, 2017
}

\begin{abstract}
This paper evaluates the performance of various measures of model-free implied volatility in predicting returns and realized volatility. The critical role of the out-of-the money call options is highlighted through an investigation of the relevance of different components of the model-free implied volatility. The Monte Carlo simulations show that: first, volatility forecasting performance of various measures can be enhanced by employing an interpolation-extrapolation technique; second, for most measures considered, gains in their predictive power for future returns can be obtained by implementing an interpolation procedure. An empirical application using SPX options recorded from 2003 to 2013 further illustrates these claims.
\end{abstract}

Keywords: Model-free implied volatility; Corridor implied volatility; Volatility forecasting; Return predictions.

JEL Classification: G17, G13, C63 


\section{Introduction}

In an efficient market, the option price embodies all available useful information about future movements of the underlying asset. Hence, traders and hedge fund managers are primarily interested in option-implied volatility when making financial decisions. As a natural forecast

of return variation over the remaining life of the relevant option, option-implied volatility has been frequently used in forecasting future volatility, see Poon \& Granger (2003) for an extensive review of the studies on this topic. As opposed to the Black-Scholes (BS) implied volatility, model-free option-implied volatilities have gained substantial popularity because, relying upon no particular parametric model, they avoid potential mis-specification problems. See, for example, Britten-Jones \& Neuberger (2000), Carr \& Wu (2006) and Taylor et al. (2010).

One of the most widely adopted measures of model-free option-implied volatility is the VIX volatility index, disseminated by the Chicago Board of Options Exchange (CBOE). The VIX provides a measure of the expected value of the $\mathrm{S} \& \mathrm{P} 500$ return variation under the risk-neutral measure and is designed to closely mimic the model-free implied volatility ( $M F I V)$. Derived by Britten-Jones \& Neuberger (2000), the MFIV is defined as an integral of cross-section of out-of-the money (OTM) European style put and call options over an infinite range of strikes for the given maturity. Jiang \& Tian (2005) show that the MFIV is a more efficient forecast for future realized volatility than the BS implied volatility and the historical realized volatility. However, Andersen \& Bondarenko (2007) argue that the $M F I V$ and VIX are biased forecasts of future volatility since they contain non-trivial and time-varying risk premiums. As a more important part of their empirical study, Andersen \& Bondarenko (2007) investigate the properties of the corridor implied volatility index $(C X)$, which is obtained from the $M F I V$ by truncating the integration domain between two barriers. Being less sensitive to variation in the market variance risk premium, the $C X$ 
with the narrowest corridor width is found to dominate other implied volatility measures in the work of Andersen \& Bondarenko (2007). Another advantage of the $C X$ is that it is constructed only over intervals of the risk-neutral density (RND) where price quotes are directly observable. By contrast, the computation requirements for deriving the $M F I V$ are not satisfied by the existing data as options are traded only over a finite range of strikes. Andersen et al. (2015) further improve the construction of the $C X$ by adopting the concept of an invariant coverage across time, which ensures that the $C X$ is coherent in the time series dimension. As compared with the VIX, which is based upon strongly time-varying coverage of the tails of the RND, the $C X$ uses a consistent range of strikes, which serves as a more accurate volatility indicator over time.

In addition to the use of implied volatilities in forecasting future volatility, prior studies also indicate that the VIX may carry some predictive power for future returns on stock market indices. For example, Giot (2005) finds that future returns are always positive (negative) for very high (low) levels of the VIX. This accords with the work of Guo \& Whitelaw (2006) who provide evidence for the positive relationship between market returns and implied volatilities. The positive relationship between the VIX and future returns is also documented in Banerjee et al. (2007) who suggest that both levels and innovations of the VIX are significantly related to future returns. That finding is indicative of a negative volatility risk premium, which is consistent with Ang et al. (2006) where stocks with high past sensitivities to the innovation in the VIX display on average future decreasing returns. The evidence that the VIX is a priced risk factor in the time series of returns helps to explain why the VIX may exhibit predictive power for future returns. Although a substantial empirical literature is devoted to the investigation of risk-return relations (see, e.g., the discussion in Rossi \& Timmermann (2010), and the many references therein), most rely on the VIX as a directly observable proxy for risk. Other measures of model-free option-implied volatility are rarely considered. 
Despite the increasing popularity of the VIX, measurement errors in its construction have been noted by Jiang \& Tian (2005). The common problem inherent in the computation of the $V I X$ as well as other measures of model-free implied volatility is that only a discrete set of strikes is actually traded in the market and that very low and high strikes are usually absent. To account for measurement errors induced by the limited number of strikes, Jiang \& Tian (2005) apply the cubic spline method to interpolate between existing strikes and exploit a flat extrapolation scheme to infer option prices beyond the truncation point. Andersen \& Bondarenko (2007) address the issue induced by the discrete set of strikes via the positive convolution approximation method proposed by Bondarenko (2003). Although interpolation and extrapolation techniques are widely accepted, it remains unclear how such techniques affect the performance of implied volatilities in predicting future returns and realized volatility. In addition, there appears to be no consensus on the roles played by the OTM call and put options in the forecast of future volatility and returns. Jackwerth (2000), Jones (2006) and Bates (2008) suggest that the OTM put options may be irrelevant to known risk factors affecting stock returns. Using a cubic spline interpolation and flat extrapolation methods, Dotsis \& Vlastakis (2016) also find that the OTM put options, especially deep OTM puts, do not contain important information with respect to equity volatility risk. They also show that the OTM call options subsume all useful information embedded in the OTM puts for forecasting future realized volatility. However, Andersen et al. (2015) show that the left tail risk, driving a substantial part of the OTM put option dynamics, exhibits strong predictive power for future excess market returns over long horizons.

Against this background, this study examines the performance of various model-free option-implied volatilities in predicting future returns and volatility and contribute to the existing literature in the following ways. First, this paper is among the first to provide simulation evidence to justify the use of the interpolation/extrapolation procedure for better forecasting performance of implied volatilities. The usefulness of this procedure is verified 
in both the simulation and empirical studies. The adoption of a stochastic volatility model with both jumps and volatility risk premium in the present study mimics more closely the observed data dynamics. This can be seen as an extension of the work of Zhang et al. (2013) where a simple square-root model of Cox et al. (1985) is employed to investigate the number of options upon the information content of the $M F I V$ in an in-sample analysis. Distinct from Zhang et al. (2013), this paper conducts comprehensive out-of-sample (OOS) volatility forecasts made by different implied volatility measures including the $M F I V$.

Second, to ascertain the relevance of the OTM call and put options, this paper considers implied volatility measures constructed entirely from the cross-section of OTM put (call) options and measures which discard the deep OTM put (call) options. This is achieved by splitting the MFIV into different components with the use of different intervals of the cross-section of OTM put and call option prices. Similar constructions of implied volatilities are conducted in Dotsis \& Vlastakis (2016) who examine the price of volatility risk in the cross-section of stock returns. With a different focus from that of Dotsis \& Vlastakis (2016), the present paper compares the fraction of the time-series variation in future returns that are explained by various measures of implied volatility. Return predictability provided by implied volatilities is investigated in the pre- and post-crisis periods, respectively. The impact of the recent financial crisis is accounted for since the crisis represents an informative period during which uncertainty and risk aversion may have been more evident than the non-crisis period, see Hilal et al. (2011) and Bates (2012).

A preview of the main findings of this study is as follows. Simulation results show that, with a wider range of strikes upon which model-free option-implied volatilities are based, the OOS volatility forecast becomes more accurate while returns tend to be less predictable. In addition, a finer partition of strikes usually leads to greater predictive power of implied volatilities for future returns. These findings warrant the application of an interpolation and extrapolation scheme in the practice of volatility forecast and an interpolation method 
only in return predictions. In the empirical study using SPX options from 2003 to 2013, the aforementioned procedure, i.e. interpolation/extrapolation methods, significantly improves the performance of different measures of implied volatility considered in the OOS volatility forecast and gives rise to higher return predictability for most measures in the post-crisis period. With the use of this procedure, the OTM SPX call options substantially dominate the OTM put options with regard to their forecasting performance. The empirical findings outlined above are in accordance with the simulation evidence. Furthermore, when measures of implied volatility are derived from the listed options only, the superiority of the OTM SPX put options over the OTM call options is noted in volatility forecast and post-crisis return predictions.

The rest of this paper is organized as follows. Section 2 provides the construction of various model-free option-implied volatility measures and realized volatility adopted in this study. Section 3 outlines the techniques adopted to address measurement errors in the construction of various implied volatilities. Section 4 presents the design and settings of the Monte Carlo study along with the results. Section 5 describes the data and Section 6 reports the empirical results. Conclusion is provided in Section 7.

\section{Construction of Volatility Measures}

This section provides an outline of the construction of various measures of volatility. Section 2.1 gives an introduction of the $M F I V$ and its components derived from OTM calls and OTM puts, respectively. The VIX index is then reviewed as a close approximation of the MFIV. Section 2.2 discusses the computation of model-free corridor implied volatilities where three different segments of the cross-section of OTM put and call option prices are adopted. Finally, in Section 2.3, the high-frequency realized volatility is defined, which is used to obtain an accurate measure of the ex-post return variation of the underlying asset. 


\subsection{Model-Free Implied Volatility and VIX}

The concept of the MFIV is derived by Britten-Jones \& Neuberger (2000). Its computation for a given maturity involves market prices for a continuum of European-style options with strikes from zero to infinity, which takes the form

$$
M F I V=\sqrt{\frac{2}{\tau} e^{r \tau}\left[\int_{0}^{F} \frac{P(\tau, K)}{K^{2}} d K+\int_{F}^{\infty} \frac{C(\tau, K)}{K^{2}} d K\right]}
$$

where $r$ is the annualized risk-free interest rate as measured by the corresponding U.S. Treasury bill rate, $\tau$ is time-to-maturity measured in annual units, $F$ is the forward price for transaction at maturity $\tau, P(\tau, K)$ and $C(\tau, K)$ are the mid-quotes for European put and call options with strike price $K$ and maturity $\tau$. By construction, only OTM options (call if $K>F$ and put otherwise) are taken into account. Motivated by Dotsis \& Vlastakis (2016), the MFIV can be further divided into two components; i.e., that from the OTM call options $(V C)$ and that from the OTM put options $(V P)$, which are given by

$$
V C=\sqrt{\frac{2}{\tau} e^{r \tau} \int_{F}^{\infty} \frac{C(\tau, K)}{K^{2}} d K}
$$

and

$$
V P=\sqrt{\frac{2}{\tau} e^{r \tau} \int_{0}^{F} \frac{P(\tau, K)}{K^{2}} d K}
$$

where $M F I V^{2}=V C^{2}+V P^{2}$.

The $V I X$ index is based on the idea of fair value of future volatility developed by Demeterfi et al. (1999), which is conceptually equivalent to the $M F I V$ in equation (1) as shown by Jiang \& Tian (2007). The general formula for computation of the VIX index is given by

$$
V I X=\sqrt{\frac{2}{\tau} \sum_{i} \frac{\Delta K_{i}}{K_{i}^{2}} e^{r \tau} Q\left(\tau, K_{i}\right)-\frac{1}{\tau}\left(\frac{F}{K_{0}}-1\right)^{2}}
$$

where $\tau=30 / 365$ is the option maturity, $K_{i}$ is the strike price of the $i$ th OTM option in the 
calculation $^{1}, K_{0}$ is the first strike price below the forward index level $F\left(K_{0} \leq F\right), Q\left(\tau, K_{i}\right)$ is the midpoint of the latest available bid and ask prices of the OTM option at strike $K_{i}$, and $\Delta K_{i}$ stands for the strike price interval as $\Delta K_{i}=\left(K_{i+1}-K_{i-1}\right) / 2$.

\subsection{Corridor Implied Volatility}

The corridor implied volatility index $(C X)$ is initially analyzed in the empirical work of Andersen \& Bondarenko (2007). Unlike the $M F I V$, which requires the availability of options with strikes from zero to infinity, the $C X$ only captures volatility over a certain segment of the underlying RND. For a fixed coverage $\left[B_{L}, B_{H}\right], 0 \leq B_{L} \leq B_{H} \leq \infty$, the $C X$ is computed as

$$
C X=\sqrt{\frac{2 e^{r \tau}}{\tau} \int_{B_{L}}^{B_{H}} \frac{M(K)}{K^{2}} d K}
$$

where the time to maturity $\tau=30$ days and $M(K)$ stands for the minimum of the put and call prices at current time such as

$$
M(K)=\min (P(\tau, K), C(\tau, K))
$$

In order to ensure an invariant portion of the strike range considered in the $C X$ across time, Andersen et al. (2015) propose the ratio $R(K)$ to determine the integration barriers of the $C X$ in equation (5) using directly observable prices of OTM call and put options only,

$$
R(K)=\frac{P(\tau, K)}{P(\tau, K)+C(\tau, K)}
$$

For given lower and upper percentiles $p, q \in(0,1), B_{L}=K_{p}=R^{-1}(p)$ and $B_{H}=K_{1-q}=$ $R^{-1}(1-q)$. In the subsequent simulation and empirical studies, three measures of the $C X$ computed from equation (5) are used where $\left[B_{L}, B_{H}\right]$ takes the values $\left[R^{-1}(0.25), R^{-1}(0.75)\right]$, $\left[R^{-1}(0), R^{-1}(0.75)\right]$ and $\left[R^{-1}(0.25), R^{-1}(1)\right]$. These implied volatilities are respectively represented by $C X N T, C X L T$ and $C X R T$. The definitions of implied volatilities considered 
in this paper are listed in Table (1). All the measures are computed from options across two nearest maturities (less than 30 days and greater than 30 days) and the 30-day implied volatilities is computed by interpolating between the two separate maturities.

\section{$2.3 \quad$ Realized Volatility}

In addition to implied volatilities, this study employs monthly realized volatility and historical volatility. A simple realized variance estimator proposed by Barndorff-Nielsen \& Shephard (2002) is employed, which is equal to the sum of intraday squared returns

$$
r v_{t}=\sum_{j=1}^{M} r_{t, j}^{2}
$$

where $r_{t, j}$ stands for intraday returns within each 5 -minute interval. The realized variance is then calculated over a period of one month in order to match the maturities of the corresponding implied volatilities

$$
R V_{t}=\frac{1}{22} \sum_{i=1}^{22} r v_{t+i}
$$

The measure $R V_{t}$ is recorded daily but contains monthly (future) variance. The substantial serial correlation induced by the construction of $R V_{t}$ in equation (8) will be accounted for in the subsequent analysis. Furthermore, the realized variance on the latest trading day, $r v_{t-1}$, is used as a proxy for historical variance, which may contain useful information for future return variation.

\section{Error Adjustment Mechanisms}

As introduced in Section 2, the $M F I V$ is computed as an integral of option prices over an infinite range of strikes; and all the measures of implied volatility that are considered require numerical integration using the trapezoidal rule. However, only a limited number of strikes 
are actually traded in the market, which may result in inaccuracies in the computation of the option-implied volatilities, so further affecting their performance in predicting future volatility and returns. Specifically, very low and high strikes are usually not available in practice, which leads to the so-called truncation errors; and the set of discrete strikes can be rather sparse, which gives rise to the discretization errors. For more details ${ }^{2}$, see Jiang \& Tian (2005). To account for the measurement errors discussed above, the use of an interpolation and extrapolation scheme is essential. The interpolation of option prices within the boundary of actual strikes is relatively straightforward, which can be carried by fitting a natural cubic spline as in the work of Jiang \& Tian (2005). The major challenge is how to extrapolate option prices towards the tails of the RND with precision. The following section provides an introduction of the extrapolation procedure adopted in the present paper.

In line with Andersen \& Bondarenko (2007), this paper estimates the RND using a nonparametric approach, the so-called positive convolution approximation (PCA) proposed by Bondarenko (2003) and then extracts prices beyond the truncation point from the estimated $\mathrm{RND}^{3}$. The PCA method for estimating the RND offers several benefits: (1) it guarantees no-arbitrage density estimates; (2) it avoids overfitting while allowing for small samples; (3) it involves simple computation algorithm only; (4) it is insensitive to the data generating process. The main idea of the PCA is to construct a set of admissible densities containing functions which can be expressed as a convolution of a fixed positive kernel and another density. The optimal density is that obtained from the admissible densities which generates the best fit to the listed option prices. The sub-section below briefly describes the RND estimation using the PCA approach.

The relationship between the RND and call/put options can be expressed as

$$
h_{0}\left(S_{\tau}\right)=\left.\frac{1}{e^{\int_{0}^{\tau} r_{s} d s}} \frac{\partial^{2} C(\tau, K)}{\partial K^{2}}\right|_{K=S_{\tau}}=\left.\frac{1}{e^{\int_{0}^{\tau} r_{s} d s}} \frac{\partial^{2} P(\tau, K)}{\partial K^{2}}\right|_{K=S_{\tau}}
$$


where $S_{\tau}$ represents the value of an underlying asset on trading date $\tau$ and $r_{s}$ is the risk-free rate. For simplicity, it is assumed that the asset pays no dividends and $r_{s}=0$ and thus $e^{\int_{0}^{\tau} r_{s} d s}=1$. In the PCA approach, the first step is to construct the approximating set $W_{h}$ representing all admissible or candidate densities, from which the optimal density is selected. Let $L^{d}$ denote the set of all probability densities, i.e., nonnegative functions that integrate to one. For a basis density $\phi(K) \in L^{d}$, a new density $\phi_{m}(K):=\frac{1}{m} \phi\left(\frac{K}{m}\right)$ can be obtained by smoothing $\phi(K)$ with the bandwidth parameter $m$. Once $\phi_{m}(K)$ is fixed, the approximating set $W_{m}=W_{\phi_{m}}$ is given by

$$
W_{m}:=\left\{g \in L^{d} \mid g=\phi_{m} * \mu, \text { for } \mu \in L^{d}\right\}
$$

which contains functions $g$, expressed as a convolution of $\phi_{m}$ with positive functions $\mu$. Although the space $L^{d}$ accommodates very general shapes of densities, $W_{m}$ is made up of only smooth and well-behaved densities. If functions $h$ (the true RND) and $g$ are both integrable, the following equation holds.

$$
h * g:=\int_{-\infty}^{\infty} h(K-y) g(y) d y
$$

An estimator of the RND is the function of $\widehat{h}(K) \in W_{m}$ which provides the best fit to a certain cross-section of options $\left\{P_{i}\right\}$, i.e. it achieves the objective function as follows

$$
\underset{\widehat{h} \in W_{m}}{\operatorname{Minimize}} \sum_{i=1}^{n}\left(P_{i}-D^{-2} \widehat{h}\left(K_{i}\right)\right)^{2}
$$

where $D^{-2} g(K)$ represents the second integral of $g(K)$ such as

$$
D^{-2} g(K):=\int_{-\infty}^{K}\left(\int_{-\infty}^{y} g(z) d z\right) d y
$$

The optimization problem in equation (12) can be solved numerically by discretizing the 
admissible set $W_{m}$ as follows

$$
\underset{\widehat{h} \in W_{m}^{\Delta z}([v, w])}{\operatorname{Minimize}} \sum_{i=1}^{n}\left(P_{i}-D^{-2} \widehat{h}\left(K_{i}\right)\right)^{2}
$$

with $\Delta z=0.5 m$, where $[v, w]$ is a large but finite interval on which the underlying density $h$ is approximated and $\Delta z$ is the grid step. Further details on the construction of the PCA estimator can be found in Bondarenko (2003). Once the estimated RND is obtained, option prices can be inferred for a continuum of strikes through the relationship in equation (9).

\section{Monte Carlo Simulation}

This section presents a Monte Carlo simulation study where different numbers of option prices are considered as the strike range and increment vary. The aim of this experiment is (i) to ascertain the impact of discrete strike prices on the performance of various implied volatility measures in forecasting future volatility and returns and (ii) to provide guidance for the use of interpolation and extrapolation technique in forecasting.

\subsection{Simulation Design}

The simulation exercise conducted in the present paper is motivated by Zhang et al. (2013) who examine the effect of the number of strikes on the information content of the $M F I V$ using a simple model without jumps and volatility risk premium. In a departure from Zhang et al. (2013), this study concentrates on the OOS volatility forecasting performance of implied volatilities and the predictive power of implied volatilities for future returns. A jump-diffusion model adopted by Duan \& Yeh (2010) is used to simulate the asset price and 
the latent stochastic volatility by

$$
\begin{aligned}
d \ln S_{t} & =\left[r-q+\delta_{s} V_{t}-\frac{V_{t}}{2}\right] d t+\sqrt{V_{t}} d W_{t}+J_{t} d N_{t}-\lambda \mu_{J} d t \\
d V_{t} & =\kappa\left(\theta-V_{t}\right) d t+v V_{t}^{\gamma} d B_{t}
\end{aligned}
$$

where $W_{t}$ and $B_{t}$ are correlated Wiener processes, having correlation coefficient equal to $\rho$; $N_{t}$ denotes a Poisson process with intensity $\lambda$, which is independent of $W_{t}$ and $B_{t} ; J_{t}$ is an independent normal random variable with mean $\mu_{J}$ and standard deviation $\sigma_{J}$. The price, $S_{t}$, and volatility, $V_{t}$, processes are dependent through the correlated diffusive terms $-W_{t}$ and $B_{t}$. The other parameters, $r, q$ and $\delta_{s}$ are the risk-free rate, the dividend yield and the asset risk premium, respectively ${ }^{4}$.

Option valuation is implemented using the corresponding model under the risk-neutral probability measure given by

$$
\begin{aligned}
d \ln S_{t} & =\left[r-q-\frac{V_{t}}{2}+\lambda^{*}\left(\mu_{J}^{*}+1-e^{\mu_{J}^{*}+\sigma_{J}^{2}}\right)\right] d t+\sqrt{V_{t}} d W_{t}^{*}+J_{t}^{*} d N_{t}^{*}-\lambda^{*} \mu_{J}^{*} d t \\
d V_{t} & =\left(\kappa \theta-\kappa^{*} V_{t}\right) d t+v V_{t}^{\gamma} d B_{t}^{*}
\end{aligned}
$$

where $\kappa^{*}=\kappa+\delta_{V}$ and $B_{t}^{*}=B_{t}+\delta_{V} / v \int_{0}^{t} V_{s}^{1-\gamma} d s$ with $\delta_{V}$ being the volatility risk premium. Again, $W_{t}^{*}$ and $B_{t}^{*}$ are the Wiener processes correlated with the coefficient $\rho ; N_{t}^{*}$ is a Poisson process with intensity $\lambda^{*}$ independent of $W_{t}^{*}$ and $B_{t}^{*}$; the independent normal random variable $J_{t}^{*}$ has a new mean $\mu_{J}^{*}$ but an unchanged standard deviation $\sigma_{J}$. The empirical martingales simulation method ${ }^{5}$ developed by Duan \& Simonato (1998) is used to compute option prices, given that there is no closed-form option pricing formula for equation (16). In addition, the theoretical $V I X$ index, represented by $V I X_{\text {Theo }}$, is computed by following equation (13) from the work of Duan \& Yeh (2010).

This study assumes one year has 252 trading days and that one day consists of 6.5 hours of open trading, as is the case on the NYSE and NASDAQ. A sparse sampling at a frequency 
of once every 5 minutes is used in this simulation study and therefore one day can be divided up into 78 intraday intervals, i.e., $\frac{6.5 \times 3600}{300}=78$. A daily series is extracted by sampling once every 78 data points. The asset price and the latent stochastic volatility are simulated according to the Euler discretized version ${ }^{6}$ of equation (15). The simulation is simplified by assuming no dividends and a zero interest rate. The initial stock price and latent stochastic volatility $^{7}$ are set respectively as 1000 and 0.08 . The sample size of daily series is 2000 . The parameter values are similar to those adopted by Duan \& Yeh (2010).

\begin{tabular}{ccccccccccccc}
\hline$\kappa$ & $\theta$ & $\lambda$ & $\mu_{J}(\%)$ & $\sigma_{J}(\%)$ & $v$ & $\rho$ & $\gamma$ & $\delta_{s}$ & $\kappa^{*}$ & $\phi^{*}(\%)$ & $\delta_{V}$ & $\delta_{J}(\%)$ \\
\hline 2.500 & 0.080 & 55.000 & 0.300 & 0.500 & 1.400 & -0.800 & 0.900 & 0.420 & -13.000 & 0.035 & -15.500 & -0.059 \\
\hline
\end{tabular}

Option prices are computed corresponding to two nearby maturities, 23 and 37 days. This experiment considers two fixed strike price increments $(\Delta K=5$ and $\Delta K=1)$ and attempts with different moneyness ranges $([0.8,1.2],[0.7,1.3]$ and $[0.6,1.4])$.

\subsection{Simulation Results}

Table (2) reports the summary statistics of various volatility measures. It is evident that the mean of the implied volatility estimates increases with the moneyness range. This accords with the work of Jiang \& Tian (2007), where the truncation errors usually result in an underestimation of the true volatility. The mean of the VIX, MFIV, CXNT, CXLT and $C X R T$ decreases as the strike increment becomes smaller, which is consistent with the finding of overestimation of the underlying volatility induced by discretization errors in Jiang \& Tian (2007). For all measures considered, the mean squared error ${ }^{8}$ (MSE) decreases with the strike range. Table (2) also shows that measures of implied volatility become more volatile with the range of strikes while they, except the $V C$, tend to appear less volatile as the partition of strikes is smaller.

To evaluate the OOS volatility forecasting performance of various option-implied volatilities, 
a univariate Mincer-Zarnowitz regression is adopted as follows

$$
y_{t+1}=\alpha_{j}+\beta_{j} x_{j, t}+\mu_{j, t+1}
$$

where $y_{t+1}$ represents the realized volatility containing the information of month $t+1$ and where $x_{j, t}$ indicates the volatility estimate $j$ among all candidate estimates. To obtain OOS forecasts of the realized volatility measure, this study employs a rolling window of 1000 observations for the one-step-ahead forecasts. The daily realized volatility in equation (8) contains substantial induced serial correlation, which seriously affects the standard errors of the coefficient estimates. To overcome this problem, the Bartlett/Newey-West heteroskedasticity consistent covariance matrix estimator with 44 lags is used, see Andersen et al. (2007). Regressions are examined for both volatility and logarithms of volatility. The forecasts are evaluated by the MSE, which is robust to the presence of noise in the volatility proxy, see Patton (2011). The OOS $R^{2}$ of the Mincer-Zarnowitz regression is also taken into account, which corrects for bias by reflecting the variance but not the bias-squared component of the MSE.

Forecasting results ${ }^{9}$ are reported in Table (3). Clearly, the VIX $X_{\text {Theo }}$ dominates all the other candidate measures in terms of the volatility forecasting performance ${ }^{10}$. Forecasting performance increases with the strike range for all the measures, except that of the $C X N T$ and $C X L T$. It is not surprising that the $C X N T$ performs the same for different moneyness ranges since the options within the barriers $B_{L}=K_{0.25}$ and $B_{H}=K_{0.75}$ are not affected by the variation in the strike range. The worse performance of the $C X L T$ with a wider range of strikes may be attributed to the poor forecasting power of the deep OTM put options for future volatility. In addition, Table (3) shows that the strike increment $\Delta K$ tends to have a negative impact on the volatility forecasting power of the $V I X, M F I V, C X N T$, $C X L T$ and $C X R T$ but exerts a positive impact on that of the $V C$ and $V P$. Overall, the 
effect of the strike range on the forecasting performance is considerable and that of the strike increment is negligible. The use of different loss functions, i.e. MSE and OOS $R^{2}$, gives the identical conclusion in terms of the role of the strike range and increment in the forecasting practice as well as the ranking of forecast performance among implied volatility candidates. These findings motivate the application of an extrapolation procedure to extend the tails of the RND in an attempt to improve the volatility forecast accuracy. On the other hand, an interpolation method is considered necessary since the number of listed options may be rather small in practice. The lack of observed options may lead to inaccuracies in the estimation of the RND using the PCA method and thus result in failure in inferring the options beyond the truncation points. Moreover, the critical role of the OTM call options is noted in Table (3) where the $V C$ serves as the top forecaster and the $C X R T$ substantially outperforms the CXLT.

The next step is to apply the natural cubic spline to interpolate between available strikes and to implement the PCA method in order to obtain the option values beyond the range of listed strikes. The corresponding measures computed by options with the use of such procedure are prefixed by $C P$-. To examine the performance of the $C P$-measures in the forecasting practice for future volatility, this study focuses on the case of $\Delta K=5$ and moneyness range $=[0.8,1.2]$ only. Specifically, a step of one unit of the index is used to numerically compute the integral in the interpolation procedure and four standard deviations from forward prices are adopted as an integration range ${ }^{11}$. The interval of strikes that are needed to extrapolate is $\left(\left[F_{0}-4 S D, K_{\min }\right]\right.$ and $\left.\left[K_{\max }, F_{0}+4 S D\right]\right)$ where $K_{\min }\left(K_{\max }\right)$ represents the minimum (maximum) listed strike price in the market. Table (4) reports the volatility forecast performance, measured by both the MSE and OOS $R^{2}$, of various implied volatility measures and their corresponding $C P$-measures. The values in parentheses below the MSE are the mean difference of squared forecasting errors between the original implied volatility measure and its corresponding $C P$-measure. Numbers in bold indicate 
statistically significant differences at $5 \%$ by the Diebold-Mariano test. Columns 1-4 show that the $C P-M F I V, C P-C X R T, C P-V C$ and $C P-V P$ achieve significant gains in the forecasting performance for future volatility and that the ranking of forecasting power of the $C P$-measures remains unchanged from that of the original measures. Columns 5-8 present values of the OOS $R^{2}$ where the percentage changes of the $R^{2}$ are represented by the numbers in parentheses and where the gains of the $C P$-measures are indicated in bold. With the single exception of $C X N T$, the use of the interpolation and extrapolation method brings higher OOS $R^{2}$ for all the measures considered.

Another important application of the implied volatility is to predict future market returns. As in the work of Banerjee et al. (2007), the 30- and 60-day future returns are regressed on daily levels ${ }^{12}$ of the implied variance estimates as follows

$$
\frac{1}{h} \sum_{j=1}^{h} r_{t+(j-1), t+j}=\alpha_{1}+\beta_{1} v_{t}+u_{t, t+h}
$$

where $v_{t}$ indicates various measures of implied variance levels. To account for residual correlation caused by overlapping returns, this study considers the Newey-West standard errors. The adjusted $R^{2}$ is employed to indicate the degree of return predictability; the values are reported in Table (5). First, results indicate that the return predictions by implied volatility measures deteriorate with the strike range. Second, with a finer partition of strikes, return predictive power generally improves, with the one exception of $V C$. From this evidence, only the interpolation method, which provides a smaller partition of strikes, is needed to achieve better return predictions by measures of implied volatility. Consistent with the work of Andersen et al. (2015), the deep OTM put options dominate the deep OTM call options in predicting future returns. This is indicated by the higher $R^{2}$ s given by the $C X L T$ relative to those by the $C X R T$. In addition, the $V C$ displays the strongest predictive power for future returns in most cases while the $V P$ serves as the top performer 
only in the case of $\Delta K=1$ when short horizon is considered. This suggests that OTM call options exhibit superior predictive power overall to that of the OTM put options for future returns. This is despite the superiority of the deep OTM puts over the deep OTM calls in this exercise.

Finally, the cubic spline is applied to achieve a finer partition of strikes in the case of return predictions. Measures of implied volatility based upon the options using the interpolation method are prefixed by $C$-. To examine the effect of the interpolation procedure on return predictions, this study takes the case of $\Delta K=5$ and moneyness range $=[0.8,1.2]$ as an example and reports the results of the return predictability in Table (6). Gains in the predictive power for future returns are only observed for $C-C X N T, C-C X L T, C-C X R T$ over 30-day and 60-day horizons, and for $C$-VP over 30-day horizon. However, given the positive impact of the strike increment on return predictions in Table (5), the interpolation procedure is expected to lead to more evident gains in the predictive power of various implied volatilities for future returns, where the partition of strikes is often much more sparse, i.e. greater than 5. Findings in Section 6 confirm this hypothesis.

\section{Data}

The data sample spans from January 02, 2003-December 31, 2013, encompassing 2769 trading days. Data are taken from several sources. Closing bid and ask SPX option prices and dividend yield are obtained from Optionmetrics via the WRDS system. High-frequency data at 5-minute intervals for the $\mathrm{SPX}^{13}$ are collected from the Tick Data Inc.. Daily one-month and three-month Treasury-bill yields ${ }^{14}$, taken as the risk-free rates, are extracted from the Federal Reserve Bulletin. In addition, the average of bid and ask is taken as the best available measure of the option price to alleviate the bid-ask bounce problem. For the two nearby maturities, there is an average of 34 out of 97 (63 out of 97) OTM call (put) 
option quotes per day. Two commonly used data filters are applied. First, options with less than seven days remaining to maturity are excluded. These options may be subject to problems of liquidity and market microstructure. Second, options violating the boundary conditions, i.e. with BS implied volatilities below zero or above $100 \%$, are excluded from the sample. Only OTM options are included since in-the-money options are less liquid and thus may induce bias into the computation of implied volatilities.

The CBOE calculates the VIX index using option prices updated every five minutes. However, the Optionmetrics database includes the last daily bid-ask quote only, which might not correspond to the data published by CBOE for their final end-of-day computation. Hence, as a more direct benchmark, this paper derives a replicated $V I X$ index, $R X$, using the exact CBOE procedure every day. Thereby, it follows the work of Andersen et al. (2015). The $R X$ provides an equivalent of the VIX using the SPX option prices from the Optionmetrics data set. It is well known that the CBOE adopts a particular rule to exclude OTM options: once two puts (calls) with consecutive strikes are found to have zero bid option prices, no puts (calls) with lower (higher) strikes are taken into account. The model-free implied volatility index with a broader strike range, denoted by $M F I V$, can be obtained by discarding any options with a zero bid price and employing all OTM options with a positive bid quote, i.e. ignoring the cutoff rule by the CBOE. Hence, the $M F I V$ provides an upper bound for $R X$. In addition, the same notations are adopted for the other candidate measures as those in the simulation study ${ }^{15}$.

For the 2769 trading days under consideration, the implied volatility measures are not available at some points due to a variety of reasons, including: (1) the requirement for the two nearby maturities is not satisfied; (2) the lack of OTM options; (3) boundary conditions are violated, which reduces the sample size to 2330 . The construction of the $R V_{t}$ leads to the loss of one month at the end. Finally, the sample data under analysis contains 2307 observations, for the period from January 02, 2003 to November 27, 2013. 


\section{Empirical Results}

This section starts by reporting the basic statistical properties of different volatility measures. It then investigates their performance as predictors of the future realized volatility and market returns of the underlying S\&P 500 index.

Table (7) reports the summary statistics ${ }^{16}$ of the monthly volatility measures which are annualized and recorded daily. First, the unconditional mean of most implied volatility measures clearly exceeds the mean of the $R V$. This is consistent with the extant literature establishing the presence of a substantial positive risk premium for bearing volatility risk. Note also that the $R V$ has the highest skewness and kurtosis statistics. This erratic nature is attributed to the unpredictable innovation term of the $R V$ as noted in the work of Andersen \& Bondarenko (2007). Second, the $C X L T(V P)$ is found to be more volatile and higher in magnitude than the $C X R T(V C)$ because deep OTM puts generally have the highest implied volatility, i.e. volatility smile. A similar phenomenon is observed in the case of the $C P$-measures. Such evidence is also given in Figure (1) which depicts the time-variation of various implied volatility candidates. In particular, the $R X$ overlaps the $M F I V$ closely and thus high similarity is expected in their forecasting power for future realized volatility and returns. Finally, all volatility measures exhibit substantial persistence with extremely slow decay in their autocorrelations.

The correlation between various measures of implied volatility and realized volatility is provided in Table (8). Compared with the measures extracted from the listed options only, the corresponding $C P$-measures display higher correlation with the $R V$. This is indicative of superior forecasting power for future volatility. Contrast to the work of Zhang et al. (2013) and Dotsis \& Vlastakis (2016) who examine the information content ${ }^{17}$ of implied volatilities in in-sample regressions, this study concentrates on their OOS volatility forecasting performances. The results of the volatility forecasts are presented in Table (9) 
where the forecasting performance is measured by the MSE and OOS $R^{2}$. Gains achieved by the $C P$-measures are generally more evident than those in the simulation study. In almost all cases, gains in MSE are significant at 5\% level. The $C X N T$ dominates other measures that are based on the existing options. The $C P-C X N T$ ranks best among all $C P$-measures. As shown in the upper panel of Table (9), CXLT (VP) outperforms the $C X R T(V C)$ in the forecasting of future volatility. This can be attributed to the fact that only a very small number of OTM calls (34 out of 97 per day on average) are available in this empirical study. However, in the lower panel, where more options are involved with the use of interpolation and extrapolation scheme, the OTM call options are superior to the OTM puts, indicated by the better forecasting performance of the $C P-C X R T(C P-V C)$ than that of the $C P-C X L T$ $(C P-V P)$. The evidence for the advantage of the OTM calls is in line with the simulation result discussed in Section 4. Moreover, conclusions drawn from Table (9) remain intact when different loss functions for OOS forecasts are considered.

Finally, the return predictability is evaluated by various implied volatilities using equation (18) where the excess returns are considered as opposed to raw returns. To better understand the predictive power of implied volatilities for future returns in different market conditions, this study further splits the data sample into pre-crisis and post-crisis periods. The beginning of the financial crisis is set at September 01, 2007. As discussed in the simulation study, only interpolation is needed in the exercise of return predictions. Values of the adjusted $R^{2}$ implied by different return regressions are reported in Table (10). In the pre-crisis period, the interpolation improves the return predictive power for 4 out of 12 measures. In the post-crisis period, this result holds for 7 out of 12 measures. Moreover, the $C$ - $V C$ dominates all the other implied volatilities in terms of the performance for predicting future returns in the post-crisis period. The $C X R T$ performs the best in such forecasting practice in the pre-crisis period. Hence, the results suggest a few good substitutes for the $V I X$ index as predictors for future returns. In the upper panel of Table (10), where measures are derived 
from the observed option prices only, the OTM call options exhibit greater predictive power for future returns than the OTM put options in the pre-crisis period while the OTM put options play a more dominant role in the post-crisis period. In the lower panel, where the cubic spline is used to interpolate between available strikes, OTM call options outperform OTM put option in predicting future returns in both pre- and post-crisis periods. This is consistent with the evidence found in the simulation study.

\section{Conclusion}

This paper examines the forecasting power of various model-free option-implied volatilities for future returns and realized volatility via both Monte Carlo simulations and an empirical study using SPX options. By decomposing the model-free implied volatility into different components using various segments of the out-of-the money (OTM) put and call options, this study ascertains the role of each of the components. The paper provides a simulation study on the impact of the strike range and increment on the predictive power of the implied volatilities. Results show that: first, the forecast accuracy for future volatility improves with the range of strikes; second, the strike range exerts a negative impact on the predictive power of the implied volatilities for future returns; third, a smaller partition of strikes tends to result in greater performance of implied volatilities in predicting returns. These findings warrant the application of an interpolation and extrapolation scheme in order to enhance the forecasting power of implied volatilities for future volatility while only an interpolation method is needed in the case of return predictions.

In both simulation and empirical studies, the superiority of the aforementioned technique, i.e. interpolation/extrapolation methods, is observed for most measures considered in forecasts of future returns and volatility. More interestingly, once this technique is implemented in the empirical case to overcome the problem of the lack of strikes, the OTM SPX call options 
clearly exhibit higher forecasting power than the OTM put options. This accords with the evidence from the simulation experiment. On the other hand, the advantages of the OTM SPX put options are noted when implied volatilities are derived from the listed options only. 


\section{Notes}

${ }^{1}$ The forward price is calculated from at-the-money options according to put-call parity, $F=K_{*}+e^{r \tau}\left[C\left(K_{*}, \tau\right)-P\left(K_{*}, \tau\right)\right]$ and $K_{*}$ is determined as the strike price for which the difference between the call and put prices is minimal. It is worth noting that, at the boundaries of strike prices, $\Delta K_{i}$ is adjusted as the difference between the two highest (or lowest ) strike prices. In addition, at the strike price $K_{0}$, the option price $Q_{i}\left(\tau, K_{i}\right)$ is modified to be the average of call and put prices. The CBOE computes the VIX from an interpolation of two volatility indices with respect to two different maturities: $\tau_{t}^{l}$ and $\tau_{t}^{u}$. The VIX index is finally obtained by taking a weighted average of these two VIX measures based on $\tau_{t}^{l}$ and $\tau_{t}^{u}$

$$
V I X=100 \times \sqrt{\left[w_{1}\left(V I X_{t}^{2}\left(\tau_{t}^{l}\right) \tau_{t}^{l}\right)+w_{2}\left(V I X_{t}^{2}\left(\tau_{t}^{u}\right) \tau_{t}^{u}\right)\right] \times \frac{365}{30}}
$$

where $w_{1}=\frac{\tau_{t}^{u}-\tau}{\tau_{t}^{u}-\tau_{t}^{l}}$ and $w_{2}=\frac{\tau-\tau_{t}^{l}}{\tau_{t}^{u}-\tau_{t}^{l}}$ so that $w_{1}+w_{2}=1$.

${ }^{2}$ Other measurement errors noted by Jiang \& Tian (2007) are widely regarded as negligible and therefore are unlikely to have any material impact on the forecasting performance of implied volatilities.

${ }^{3}$ Different ways of interpolation and extrapolation were attempted in this study. For example, the clamped cubic spline interpolation and the smoothing spline delivered results similar to those based on the natural cubic spline technique. In addition, attempts were made to extend the tails of the RND using the flat-line extrapolation and to approximate the tails of the RND following a generalized extreme value distribution. These methods are dominated by the use of the PCA approach as introduced in the main text.

${ }^{4}$ The mean of $J_{t} d N_{t}-\lambda \mu_{J} d t$ is zero due to the introduction of the term $\lambda \mu_{J} d t$, which serves to center the Poisson innovation.

${ }^{5}$ The simulation sample path is set to 1000 . Put option prices are computed through put-call parity.

${ }^{6}$ The asset price and volatility path will be discretized into constant-increment time steps of $\Delta t=\frac{1}{78 \times 252}$. The discretization for the price and volatility processes through Euler scheme 
is given by

$$
\begin{aligned}
& S_{i+1}=S_{i} \exp \left[\left(r-q+\delta_{s} V_{i}-0.5 V_{i}\right) \Delta t+\sqrt{V_{i} \Delta t} W_{t}+J_{t} N_{t}-\lambda \mu_{J} \Delta t\right] \\
& V_{i+1}=V_{i}+\left(\kappa \theta-\kappa V_{i}\right) \Delta t+\rho v V_{i}^{\gamma} \sqrt{\Delta t} W_{t}+\sqrt{1-\rho^{2}} v V_{i}^{\gamma} \sqrt{\Delta t} B_{t}
\end{aligned}
$$

where $J_{t}$ is i.i.d. $N\left(\mu_{J}, \sigma_{J}\right), N_{t}$ is Poiss $(\lambda \Delta t), W_{t}$ and $B_{t}$ are two Brownian-motion processes, and $\rho$ represents the instantaneous correlation between the return process and the volatility process. As introduced in the main text, $S_{0}=1000$ and $V_{0}=0.08$.

${ }^{7}$ This study also considers a low volatility setting by letting the initial latent stochastic volatility equal 0.02. Conclusions remain unchanged.

${ }^{8}$ This is defined as the time-series average of the squared differences between the certain volatility estimate and the theoretical VIX index, VIX $X_{\text {Theo }}$.

${ }^{9}$ Motivated by the study of Andersen et al. (2015), this study also attempts to construct the realized variance using the sum of a weighted average of the log and simple squared returns, represented by $R V^{w}$. Values of the MSE for the forecasts of $R V^{w}$ remain virtually unchanged.

${ }^{10}$ In several situations, the $r v_{t-1}$ outperforms option-implied volatility estimates, which seems to contradict the findings of Jiang \& Tian (2005) and Andersen \& Bondarenko (2007). The explanation is that this experiment considers the case of very high volatility, i.e. $V_{0}=$ 0.08. When the initial latent stochastic volatility is set lower, the performance of daily lagged $R V$ falls as compared with the other implied volatility measures.

${ }^{11}$ The choice of the truncation point is motivated by the finding of Jiang \& Tian (2005) who show that the truncation errors are virtually zero beyond $3.5 S D$ from $F_{0}$.

${ }^{12}$ As a robustness check, the analysis of return predictions is also conducted by regressing future returns on the innovations of the implied variances, motivated by the work of Banerjee et al. (2007). Conclusions remain unchanged.

${ }^{13}$ In order to measure the return variation during the overnight period, the squared overnight returns, computed as the squared close-to-open logarithmic price change, are added to the realized variance obtained over the trading day.

${ }^{14}$ Following the work of Jiang \& Tian (2007), the risk-free rate is linearly interpolated 
between these two yields. However, when the maturity is shorter (longer) than one (three) month, the one-month (three-month) yield is adopted.

${ }^{15}$ Throughout the empirical work, this paper makes use of the robust forward $F$ as in the work of Andersen et al. (2015) rather than the "implied" forward $F$ determined by the CBOE according to put-call parity. However, the CBOE $F$ is still employed in computing the $R X$ in order to approximate the VIX.

${ }^{16}$ In the empirical study, the $M F I V$ is computed in the same way as the CBOE VIX in equation (4) but it ignores the cutoff rule by the CBOE. The $V C$ and $V P$ are computed as equations (2) and (3). This explains why $M F I V^{2} \neq V C^{2}+V P^{2}$ in Table (7). The reason for the use of the CBOE computation procedure, instead of the traditional MFIV calculatoin method, is that the latter results in poorer forecasting performance compared with the $R X$. All the other measures are computed in the same way as in our simulation study. The results for the $M F I V$ constructed by equation (1) can be obtained from the author upon request.

${ }^{17}$ This paper also conducts in-sample regressions to examine the volatility forecasting ability of various measures of implied volatility. Results show that the interpolation and extrapolation procedure largely improves the explanatory power of the implied volatilities in all cases. For the sake of brevity, results are not reported but can be obtained from the author upon request. 


\section{References}

Andersen, T., Bollerslev, T., \& Diebold, F. (2007). Roughing it up: Including jump components in the measurement, modeling, and forecasting of return volatility. The Review of Economics and Statistics, 89, 701-720.

Andersen, T. G. \& Bondarenko, O. (2007). Construction and interpretation of model-free implied volatility. In I. Nelken (Ed.), Volatility as an Asset Class (pp. 141-181). London, UK.

Andersen, T. G., Bondarenko, O., \& Gonzalez-Perez, M. T. (2015). Exploring return dynamics via corridor implied volatility. The Review of Financial Studies, 28, 2902-2945.

Andersen, T. G., Fusari, N., \& Todorov, V. (2015). The risk premia embedded in index options. Journal of Financial Economics, 117, 558-584.

Ang, A., Hodrick, R. J., Xing, Y., \& Zhang, X. (2006). The cross-section of volatility and expected returns. Journal of Finance, 61, 259-299.

Banerjee, P. S., Doran, J. S., \& Peterson, D. R. (2007). Implied volatility and future portfolio returns. Journal of Banking and Finance, 31, 3183-3199.

Barndorff-Nielsen, O. E. \& Shephard, N. (2002). Econometric analysis of realized volatility and its use in estimating stochastic volatility models. Journal of the Royal Statistical Society. Series B: Statistical Methodology, 64, 253-280.

Bates, D. S. (2008). The market for crash risk. Journal of Economic Dynamics and Control, $32,2291-2321$.

Bates, D. S. (2012). U.S. stock market crash risk, 1926-2010. Journal of Financial Economics, 105, 229-259. 
Bondarenko, O. (2003). Estimation of risk-neutral densities using positive convolution approximation. Journal of Econometrics, 116, 85-112.

Britten-Jones, M. \& Neuberger, A. (2000). Option prices, implied price processes, and stochastic volatility. Journal of Finance, 55, 839-866.

Carr, P. \& Wu, L. (2006). A tale of two indices. The Journal of Derivatives, 13, 13-29.

Cox, J. C., Ingersoll, J. E., \& Ross, S. A. (1985). A theory of the term structure of interest rates. Econometrica, 53, 385-407.

Demeterfi, K., Derman, E., Kamal, M., \& Zou, J. (1999). More than you ever wanted to know about volatility swaps. In Quantitative Strategies Research Notes. Goldman Sachs.

Dotsis, G. \& Vlastakis, N. (2016). Corridor volatility risk and expected returns. Journal of Futures Markets, 36, 488-505.

Duan, J.-C. \& Simonato, J.-G. (1998). Empirical martingale simulation for asset prices. Management Science, 44, 1218-1233.

Duan, J.-C. \& Yeh, C.-Y. (2010). Jump and volatility risk premiums implied by VIX. Journal of Economic Dynamics and Control, 34, 2232-2244.

Giot, P. (2005). Relationships between implied volatility indexes and stock index returns. The Journal of Portfolio Management, 31, 92-100.

Guo, H. \& Whitelaw, R. F. (2006). Uncovering the risk-return relation in the stock market. The Journal of Finance, 61, 1433-1463.

Hilal, S., Poon, S.-h., \& Tawn, J. (2011). Hedging the black swan: conditional heteroskedasticity and tail dependence in S\&P 500 and VIX. Journal of Banking and Finance, 35, 2374-2387. 
Jackwerth, J. C. (2000). Recovering risk aversion from option prices and realized returns. The Review of Financial Studies, 13, 433-451.

Jiang, G. J. \& Tian, Y. S. (2005). The model-free implied volatility and its information content. The Review of Financial Studies, 18, 1305-1342.

Jiang, G. J. \& Tian, Y. S. (2007). Extracting model-free volatility from option prices: an examination of the VIX index. Journal of Derivatives, 14, 35-60.

Jones, C. S. (2006). A nonlinear factor analysis of S\&P 500 index option returns. The Journal of Finance, 61, 2325-2363.

Patton, A. J. (2011). Volatility forecast comparison using imperfect volatility proxies. Journal of Econometrics, 160, 246-256.

Poon, S.-H. \& Granger, C. (2003). Forecasting volatility in financial markets: a review. Journal of Economic Literature, 41, 478-539.

Rossi, A. \& Timmermann, A. (2010). What is the shape of the risk-return relation? In $A F A$ 2010 Atlanta Meetings Paper, volume 12, (pp. 89-110).

Taylor, S. J., Yadav, P. K., \& Zhang, Y. (2010). The information content of implied volatilities and model-free volatility expectations: evidence from options written on individual stocks. Journal of Banking and Finance, 34, 871-881.

Zhang, Y., Taylor, S. J., \& Wang, L. (2013). Investigating the information content of the model-free volatility expectation by monte carlo methods. Journal of Futures Markets, 33, 1071-1095. 


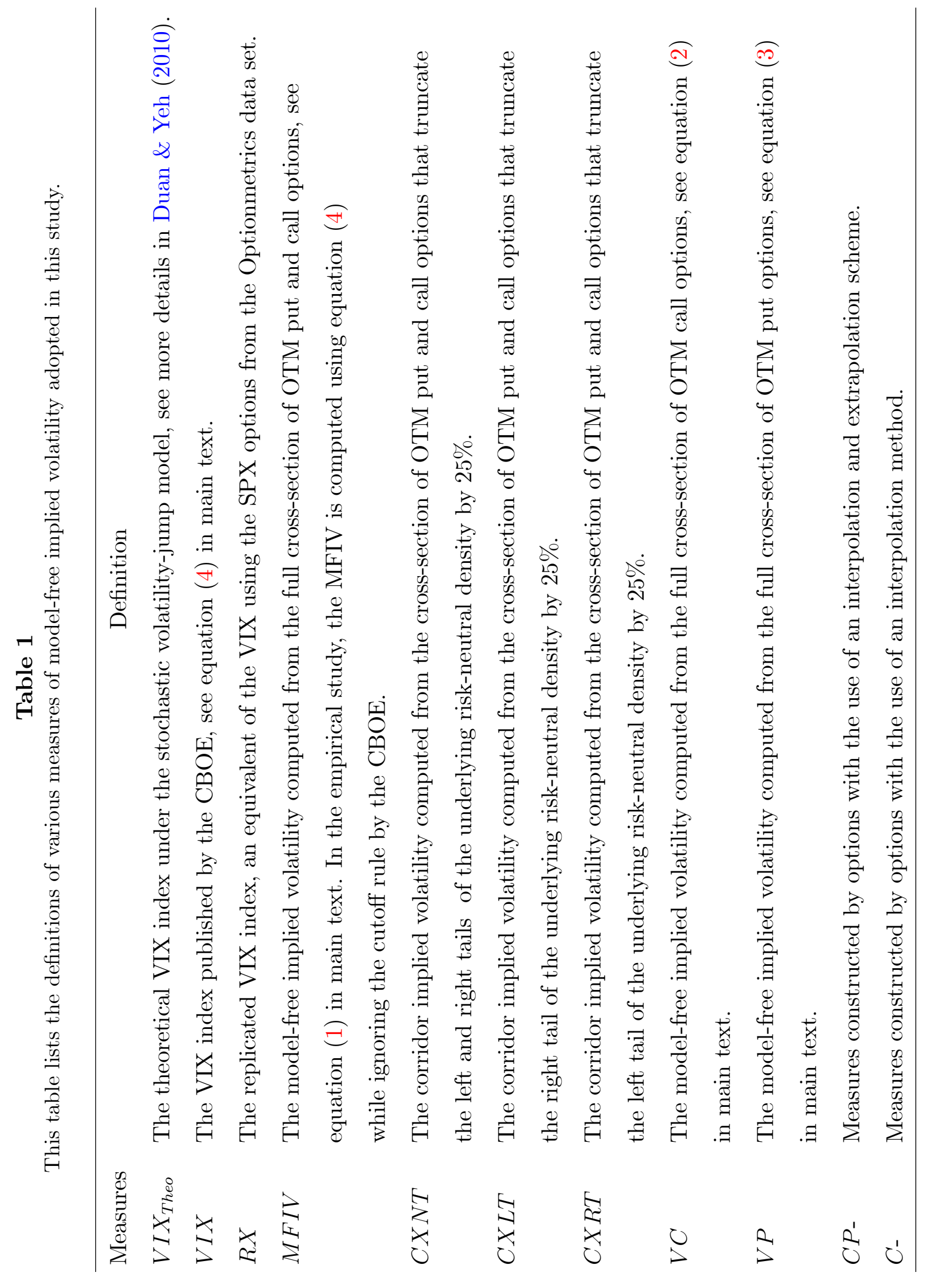




\section{Table 2}

Simulation study: summary Statistics. This table reports the mean, standard deviation, lower quartile (25\%), median (50\%), and upper quartile (75\%) of daily annualized volatility estimates over 2000 days. All the values are percentages. The mean squared estimation error, MSE, is the average of the squared differences between the volatility estimates and the theoretical $V I X$ index, $V I X^{T h e o}$. The strike price increment is denoted by $\Delta K$ and $N_{K}$ refers to the number of available options on each estimation day.

\begin{tabular}{|c|c|c|c|c|c|c|c|c|c|}
\hline \multirow{4}{*}{$\Delta K=5$} & $\begin{array}{l}V I X_{\text {Theo }} \\
R V\end{array}$ & & \multirow[b]{2}{*}{ Moneyness Range } & $\begin{array}{c}\text { Mean } \\
39.1297 \\
23.6841\end{array}$ & $\begin{array}{c}\text { StdDev } \\
14.3567 \\
9.4726\end{array}$ & $\begin{array}{c}25 \% \\
29.4363 \\
17.2824\end{array}$ & $\begin{array}{c}50 \% \\
35.3602 \\
21.5069\end{array}$ & $\begin{array}{c}75 \% \\
43.8213 \\
26.7618\end{array}$ & MSE \\
\hline & $V I X$ & & & 36.2688 & 113866 & 28.1721 & 33.8260 & 414539 & 291182 \\
\hline & & 128 & {$[0.7,1.3]$} & 37.7167 & 12.9644 & 28.6248 & 34.6614 & 43.2231 & 16.8826 \\
\hline & & 167 & {$[0.6,1.4]$} & 38.2435 & 13.8219 & 28.6925 & 34.8117 & 43.7395 & 14.2855 \\
\hline & $M F I V$ & 86 & {$[0.8,1.2]$} & 36.9711 & 11.3161 & 28.8961 & 34.5055 & 42.2275 & 25.6308 \\
\hline & & 128 & {$[0.7,1.3]$} & 38.6101 & 13.0396 & 29.4648 & 35.5447 & 44.2569 & 14.7022 \\
\hline & & 167 & {$[0.6,1.4]$} & 39.2324 & 13.9959 & 29.5486 & 35.7811 & 44.8681 & 13.1674 \\
\hline & $C X N T$ & 86 & {$[0.8,1.2]$} & 27.9266 & 10.2982 & 20.9701 & 25.4019 & 31.7878 & 150.4968 \\
\hline & & 128 & {$[0.7,1.3]$} & 27.9266 & 10.2982 & 20.9701 & 25.4019 & 31.7878 & 150.4968 \\
\hline & & 167 & {$[0.6,1.4]$} & 27.9266 & 10.2982 & 20.9701 & 25.4019 & 31.7878 & 150.4968 \\
\hline & $C X L T$ & 86 & {$[0.8,1.2]$} & 34.2586 & 10.9680 & 26.5616 & 31.8153 & 39.0299 & 47.3269 \\
\hline & & 128 & {$[0.7,1.3]$} & 35.6924 & 12.3049 & 27.0270 & 32.7474 & 40.9577 & 30.1992 \\
\hline & & 167 & {$[0.6,1.4]$} & 36.2805 & 13.1204 & 27.1798 & 32.9743 & 41.6377 & 24.6749 \\
\hline & $C X R T$ & 86 & {$[0.8,1.2]$} & 31.2108 & 10.6210 & 23.8673 & 28.6452 & 35.5908 & 84.5573 \\
\hline & & 128 & $0.7,1.3$ & 31.5717 & 11.1677 & 23.8923 & 28.7841 & 36.0049 & 74.6089 \\
\hline & & 167 & {$[0.6,1.4]$} & 31.6768 & 11.4019 & 23.8933 & 28.8077 & 36.0561 & 71.2892 \\
\hline & $V C$ & 86 & {$[0.8,1.2]$} & 22.9491 & 7.2992 & 17.7665 & 21.2982 & 26.0928 & 316.1882 \\
\hline & & 128 & $0.7,1.3$ & 23.4417 & 8.0621 & 17.8222 & 21.4521 & 26.5423 & 289.5784 \\
\hline & & 167 & {$[0.6,1.4]$} & 23.5835 & 8.3822 & 17.8222 & 21.4761 & 26.6604 & 280.8594 \\
\hline & $V P$ & 86 & {$[0.8,1.2]$} & 28.4255 & 8.5915 & 22.2635 & 26.6314 & 32.5155 & 161.1815 \\
\hline & & 128 & {$[0.7,1.3]$} & 30.1422 & 10.2282 & 22.8413 & 27.8142 & 34.7325 & 113.4245 \\
\hline & & 167 & {$[0.6,1.4]$} & 30.8299 & 11.1983 & 23.0182 & 28.0057 & 35.4366 & 95.2843 \\
\hline & $V I X$ & 422 & {$[0.8,1.2]$} & 36.1206 & 11.2236 & 28.1317 & 33.7444 & 41.3216 & 31.0087 \\
\hline & & 629 & {$[0.7,1.3]$} & 37.6563 & 12.8712 & 28.6170 & 34.6308 & 43.1692 & 17.3348 \\
\hline & & 1239 & {$[0.6,1.4]$} & 38.2166 & 13.7685 & 28.6902 & 34.8077 & 43.7299 & 14.3939 \\
\hline & $M F I V$ & 422 & {$[0.8,1.2]$} & 36.8572 & 11.1951 & 28.8665 & 34.4344 & 42.1000 & 26.8896 \\
\hline & & 629 & {$[0.7,1.3]$} & 38.5635 & 12.9668 & 29.4599 & 35.5384 & 44.2100 & 14.9503 \\
\hline & & 1239 & {$[0.6,1.4]$} & 39.2117 & 13.9525 & 29.5504 & 35.7789 & 44.8443 & 13.1920 \\
\hline & $C X N T$ & 422 & {$[0.8,1.2]$} & 27.6792 & 10.2485 & 20.7987 & 25.1750 & 31.5773 & 156.4750 \\
\hline & & 629 & {$[0.7,1.3]$} & 27.6792 & 10.2485 & 20.7987 & 25.1750 & 31.5773 & 156.4750 \\
\hline & & 1239 & {$[0.6,1.4]$} & 27.6792 & 10.2485 & 20.7987 & 25.1750 & 31.5773 & 156.4750 \\
\hline & $C X L T$ & 422 & {$[0.8,1.2]$} & 34.0638 & 10.8394 & 26.4723 & 31.6732 & 38.8431 & 50.1150 \\
\hline & & 629 & {$[0.7,1.3]$} & 35.5644 & 12.2193 & 26.9314 & 32.6610 & 40.8070 & 31.4616 \\
\hline & & 1239 & {$[0.6,1.4]$} & 36.1791 & 13.0634 & 27.0938 & 32.8640 & 41.5633 & 25.4219 \\
\hline & $C X R T$ & 422 & {$[0.8,1.2]$} & 31.0706 & 10.5707 & 23.7608 & 28.5266 & 35.5119 & 87.1688 \\
\hline & & 629 & {$[0.7,1.3]$} & 31.4414 & 11.1297 & 23.8061 & 28.6810 & 35.9053 & 76.8183 \\
\hline & & 1239 & {$[0.6,1.4]$} & 31.5491 & 11.3691 & 23.8061 & 28.6948 & 35.9247 & 73.3751 \\
\hline & $V C$ & 422 & {$[0.8,1.2]$} & 23.2036 & 7.3257 & 18.0626 & 21.5130 & 26.4031 & 307.5802 \\
\hline & & 629 & $0.7,1.3]$ & 23.7030 & 8.0965 & 18.0836 & 21.6831 & 26.9452 & 280.9600 \\
\hline & & 1239 & {$[0.6,1.4]$} & 23.8465 & 8.4200 & 18.0880 & 21.6981 & 27.0022 & 272.2445 \\
\hline & $V P$ & 422 & {$[0.8,1.2]$} & 28.5067 & 8.5052 & 22.4153 & 26.7591 & 32.5390 & 160.2375 \\
\hline & & 629 & {$[0.7,1.3]$} & 30.2902 & 10.1843 & 23.0286 & 28.0615 & 34.8485 & 110.9583 \\
\hline & & 1239 & {$[0.6,1.4]$} & 31.0050 & 11.1826 & 23.1910 & 28.3104 & 35.7387 & 92.3282 \\
\hline
\end{tabular}




\section{Table 3}

Simulation study: out-of-sample forecast losses. This table reports the ratio of the losses (MSE and $R^{2}$ ) for different predictive regressions for future monthly realized volatility and logarithm of volatility, respectively. Different strike price increments and ranges of strikes are considered here. Data are obtained for every trading day and the forecasts are based on re-estimating the parameters of the different regressions each day with a fixed length Rolling Window $(R W)$ made up of the previous 1000 days. Ranking is obtained for different cases of strike increments and represents the average volatility forecasting performances of implied volatilities across different strike ranges.

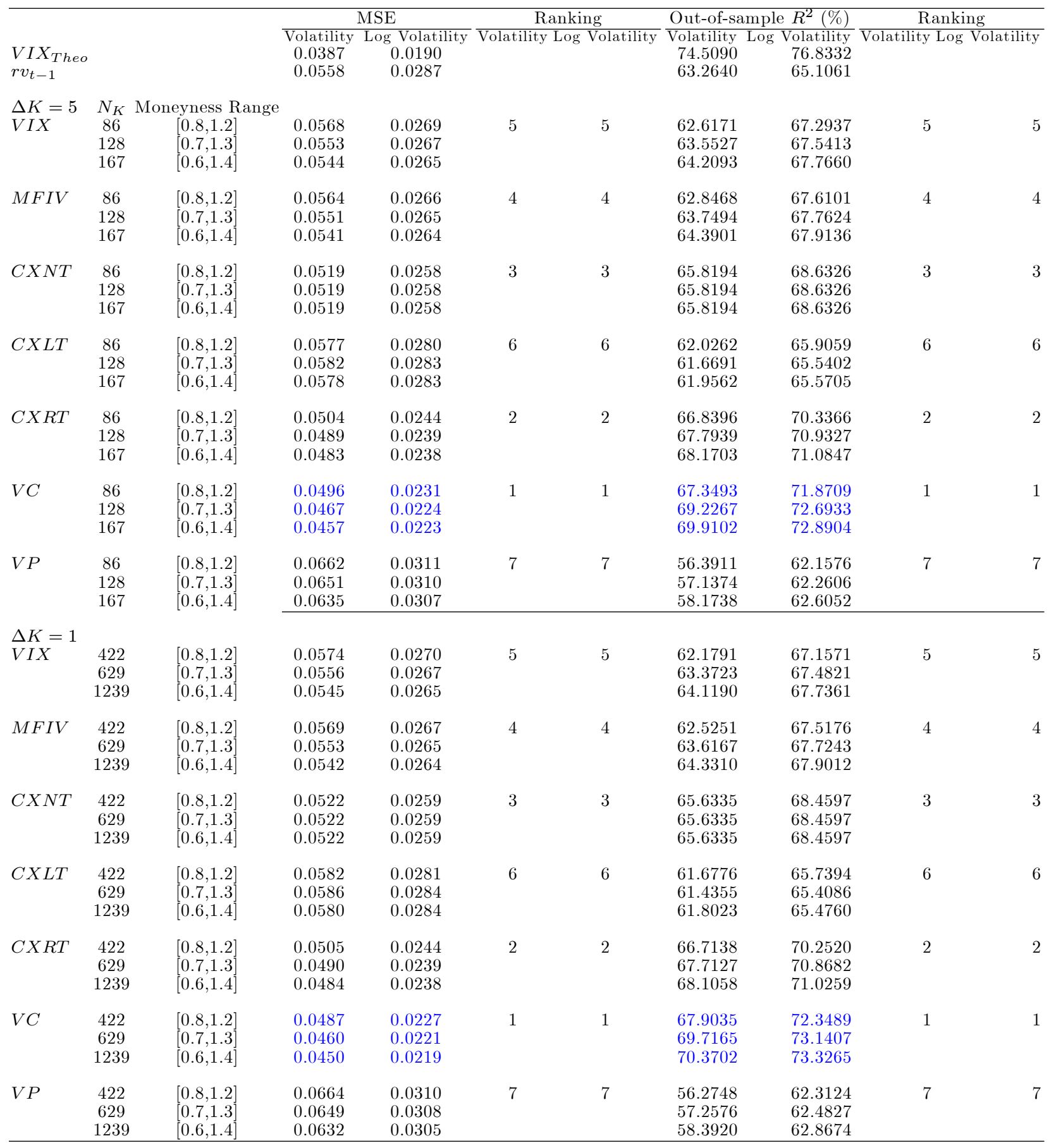




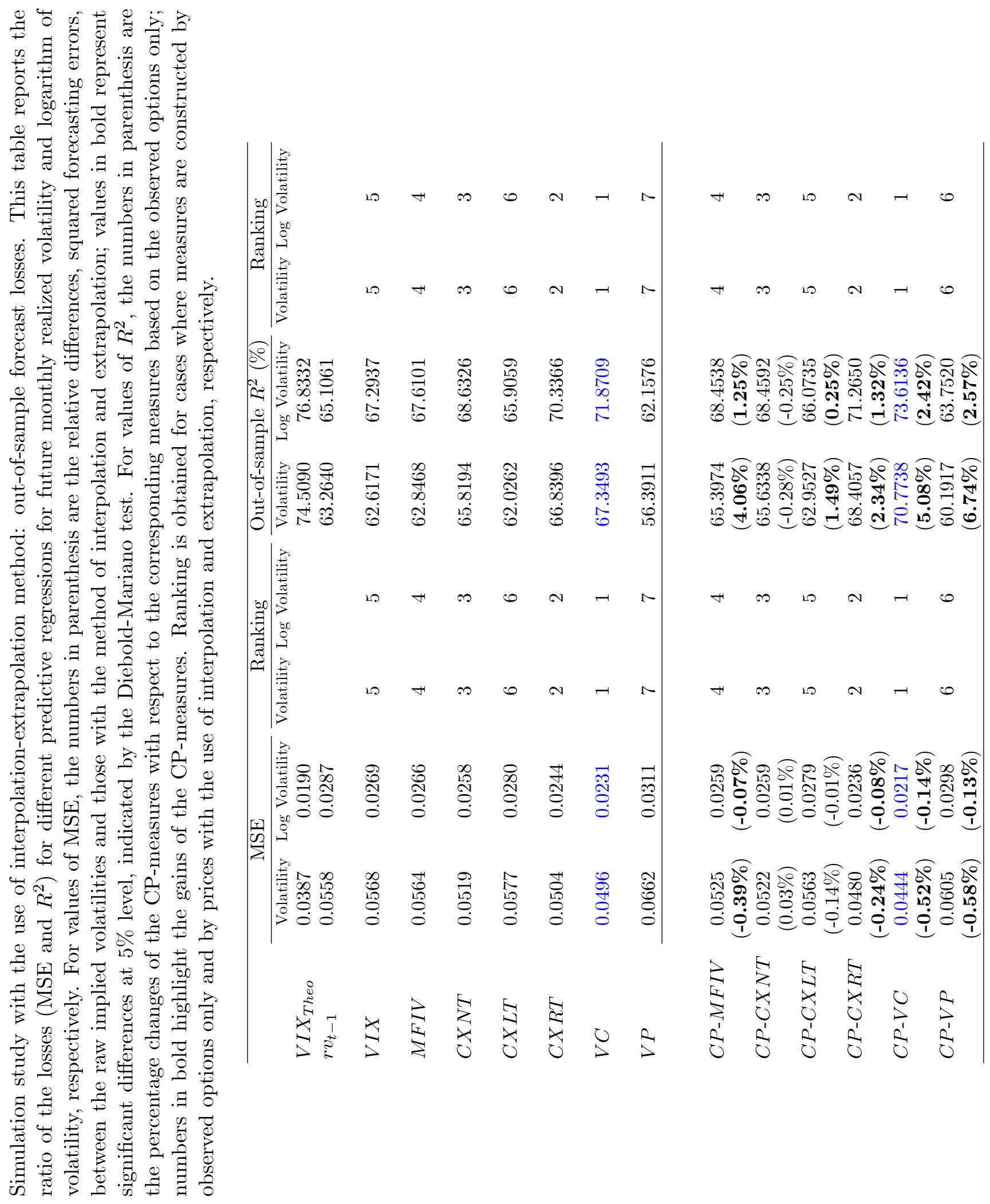




\section{Table 5}

Simulation study: multi-period return prediction. This table shows the adjusted $R^{2}$ from the daily regressions of the $h$-period returns on the current implied variance levels. Ranking is obtained for different cases of strike increments and represents the average ability of implied volatilities for predicting returns across different strike ranges.

\begin{tabular}{|c|c|c|c|c|c|c|c|}
\hline \multirow{3}{*}{$\Delta K=5$} & \multirow[b]{2}{*}{$V I X_{\text {Theo }}$} & & \multirow{3}{*}{ Moneyness Range } & \multicolumn{2}{|c|}{ Return Predictability (Adj $R^{2} \%$ ) } & \multicolumn{2}{|c|}{ Ranking } \\
\hline & & & & $\begin{array}{l}\text { 30-day } \\
0.7611\end{array}$ & $\begin{array}{l}60-\text { day } \\
7.4725\end{array}$ & 30-day & 60-day \\
\hline & \multirow{3}{*}{$V I X$} & & & 14022 & 85224 & \multirow{3}{*}{4} & \multirow{3}{*}{4} \\
\hline & & 128 & $0.7,1.3$ & 1.2048 & $\begin{array}{l}0.0224 \\
8.3069\end{array}$ & & \\
\hline & & 167 & {$[0.6,1.4]$} & 1.0217 & 8.0018 & & \\
\hline & \multirow[t]{3}{*}{$M F I V$} & 86 & {$[0.8,1.2]$} & 1.4689 & 8.6001 & \multirow[t]{3}{*}{2} & \multirow[t]{3}{*}{3} \\
\hline & & 128 & {$[0.7,1.3]$} & 1.2625 & 8.3965 & & \\
\hline & & 167 & {$[0.6,1.4]$} & 1.0648 & 8.0852 & & \\
\hline & \multirow[t]{3}{*}{$C X N T$} & 86 & {$[0.8,1.2]$} & 0.7559 & 7.4092 & \multirow[t]{3}{*}{7} & \multirow[t]{3}{*}{7} \\
\hline & & 128 & {$[0.7,1.3]$} & 0.7559 & 7.4092 & & \\
\hline & & 167 & {$[0.6,1.4]$} & 0.7559 & 7.4092 & & \\
\hline & \multirow[t]{3}{*}{$C X L T$} & 86 & {$[0.8,1.2]$} & 1.1687 & 8.1426 & \multirow[t]{3}{*}{5} & \multirow[t]{3}{*}{5} \\
\hline & & 128 & {$[0.7,1.3]$} & 1.0992 & 8.0754 & & \\
\hline & & 167 & {$[0.6,1.4]$} & 0.9731 & 7.8621 & & \\
\hline & \multirow[t]{3}{*}{$C X R T$} & 86 & {$[0.8,1.2]$} & 1.0860 & 8.1113 & \multirow[t]{3}{*}{6} & \multirow[t]{3}{*}{6} \\
\hline & & 128 & {$[0.7,1.3]$} & 0.9967 & 7.9809 & & \\
\hline & & 167 & {$[0.6,1.4]$} & 0.9164 & 7.8265 & & \\
\hline & \multirow[t]{3}{*}{$V C$} & 86 & {$[0.8,1.2]$} & 1.6091 & 8.8549 & \multirow[t]{3}{*}{1} & 1 \\
\hline & & 128 & {$[0.7,1.3]$} & 1.3245 & 8.5471 & & \\
\hline & & 167 & {$[0.6,1.4]$} & 1.1319 & 8.2404 & & \\
\hline & $V P$ & 86 & {$[0.8,1.2]$} & 1.4520 & 8.6069 & 3 & 2 \\
\hline & & 128 & $0.7,1.3]$ & 1.2745 & 8.4381 & & \\
\hline & & 167 & {$[0.6,1.4]$} & 1.0611 & 8.1023 & & \\
\hline$\Delta K=1$ & $V I X$ & 422 & {$[0.8,1.2]$} & 1.4875 & 8.6919 & 4 & 4 \\
\hline & & 629 & $0.7,1.3]$ & 1.2448 & 8.3953 & & \\
\hline & & 1239 & {$[0.6,1.4]$} & 1.0541 & 8.0624 & & \\
\hline & $M F I V$ & 422 & {$[0.8,1.2]$} & 1.5257 & 8.7259 & 2 & 2 \\
\hline & & 629 & {$[0.7,1.3]$} & 1.2881 & 8.4596 & & \\
\hline & & 1239 & {$[0.6,1.4]$} & 1.0907 & 8.1320 & & \\
\hline & $C X N T$ & 422 & {$[0.8,1.2]$} & 0.7798 & 7.4631 & 7 & 7 \\
\hline & & 629 & {$[0.7,1.3]$} & 0.7798 & 7.4631 & & \\
\hline & & 1239 & {$[0.6,1.4]$} & 0.7798 & 7.4631 & & \\
\hline & $C X L T$ & 422 & {$[0.8,1.2]$} & 1.2324 & 8.2971 & 5 & 5 \\
\hline & & 629 & {$[0.7,1.3]$} & 1.1336 & 8.1630 & & \\
\hline & & 1239 & {$[0.6,1.4]$} & 1.0068 & 7.9322 & & \\
\hline & $C X R T$ & 422 & {$[0.8,1.2]$} & 1.1023 & 8.1470 & 6 & 6 \\
\hline & & 629 & {$[0.7,1.3]$} & 1.0128 & 8.0122 & & \\
\hline & & 1239 & {$[0.6,1.4]$} & 0.9320 & 7.8527 & & \\
\hline & $V C$ & 422 & {$[0.8,1.2]$} & 1.4980 & 8.8190 & 3 & 1 \\
\hline & & 629 & {$[0.7,1.3]$} & 1.2473 & 8.5171 & & \\
\hline & & 1239 & {$[0.6,1.4]$} & 1.0707 & 8.2103 & & \\
\hline & $V P$ & 422 & {$[0.8,1.2]$} & 1.5611 & 8.6914 & 1 & 3 \\
\hline & & 629 & $0.7,1.3]$ & 1.3228 & 8.4411 & & \\
\hline & & 1239 & {$[0.6,1.4]$} & 1.1071 & 8.0958 & & \\
\hline
\end{tabular}




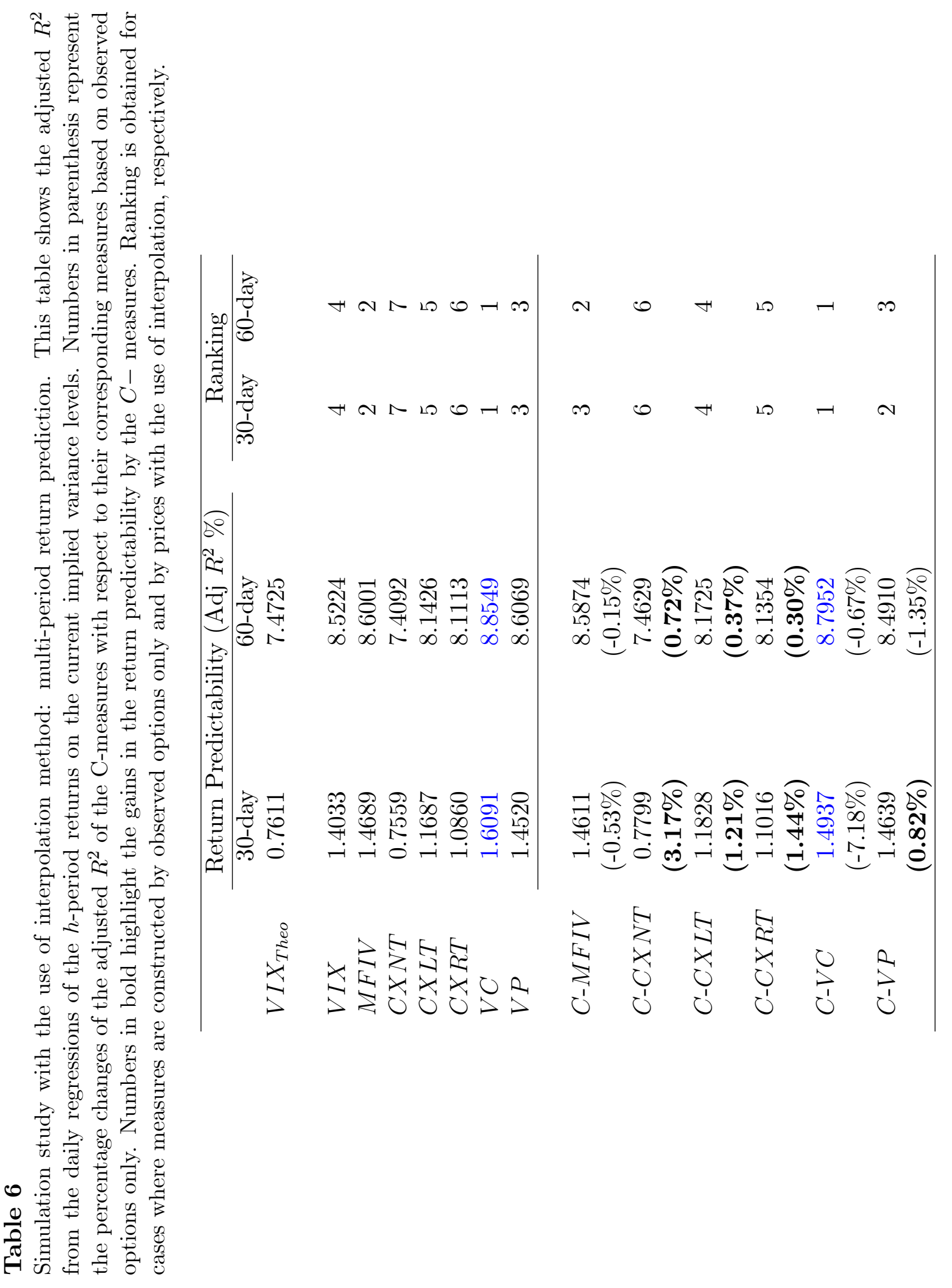




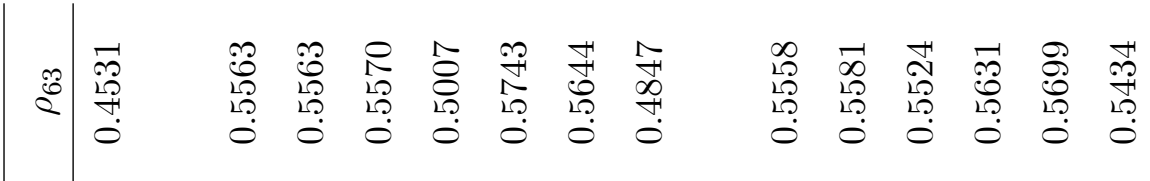

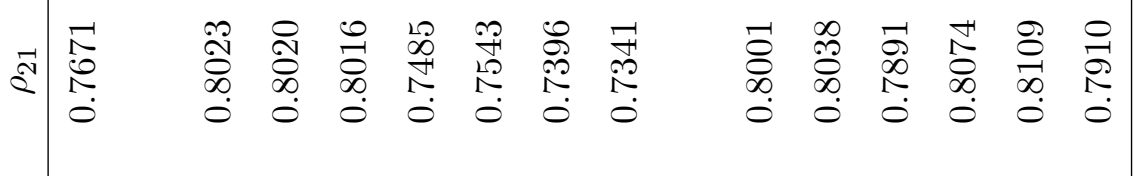

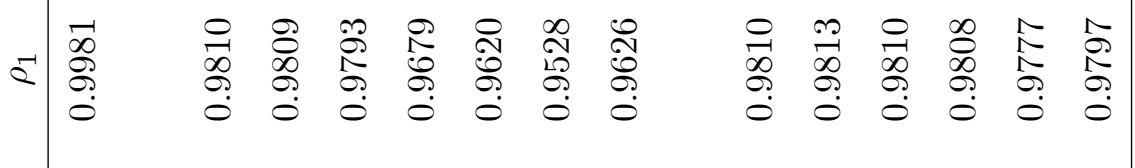




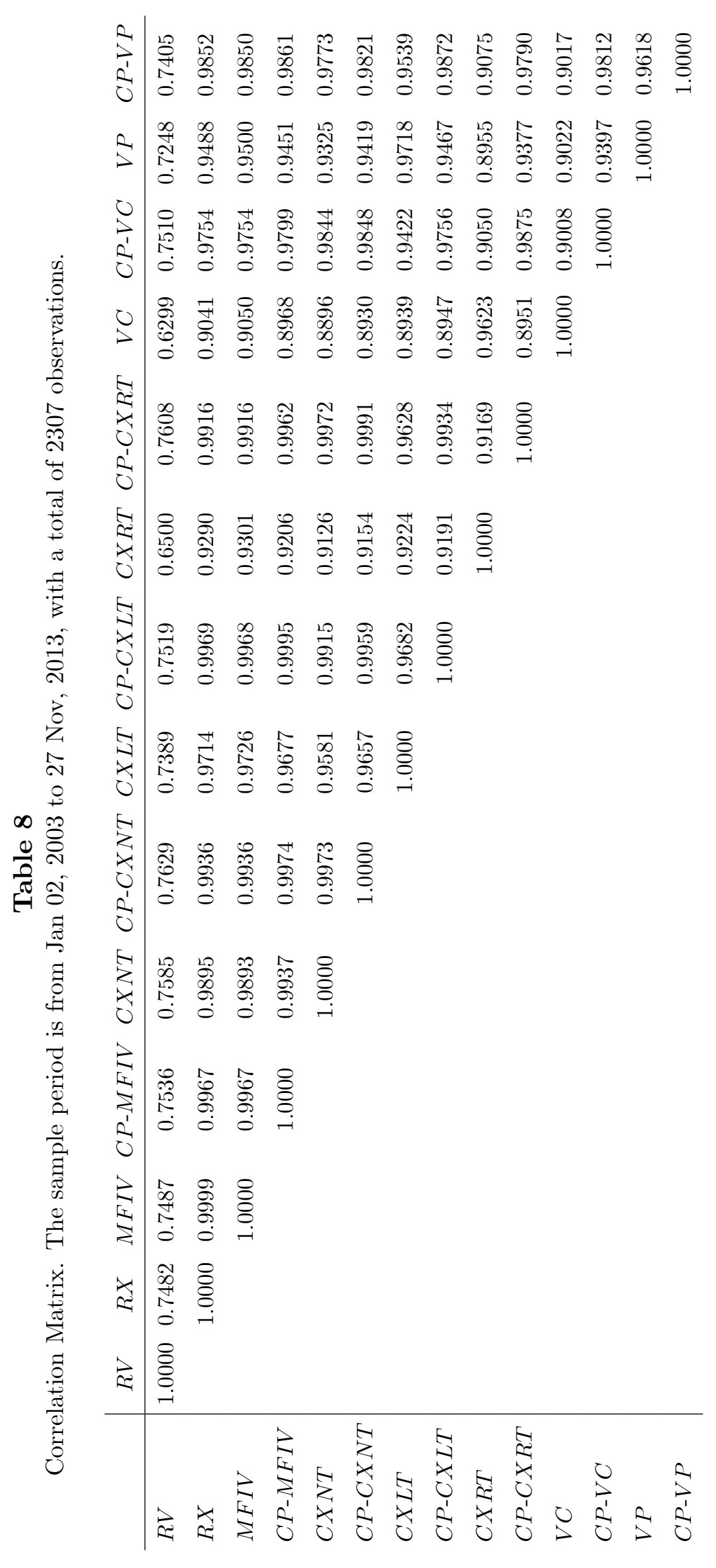




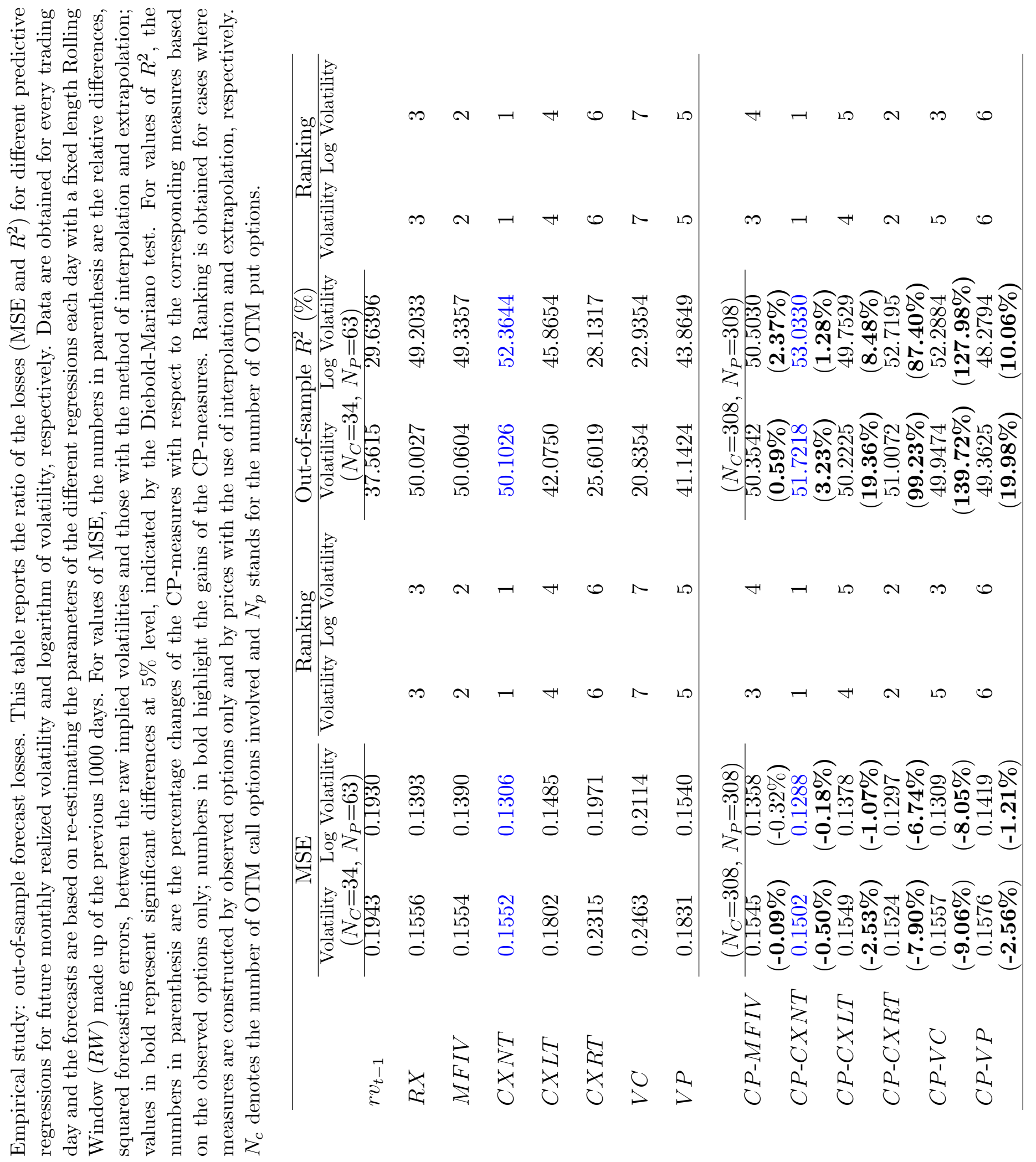




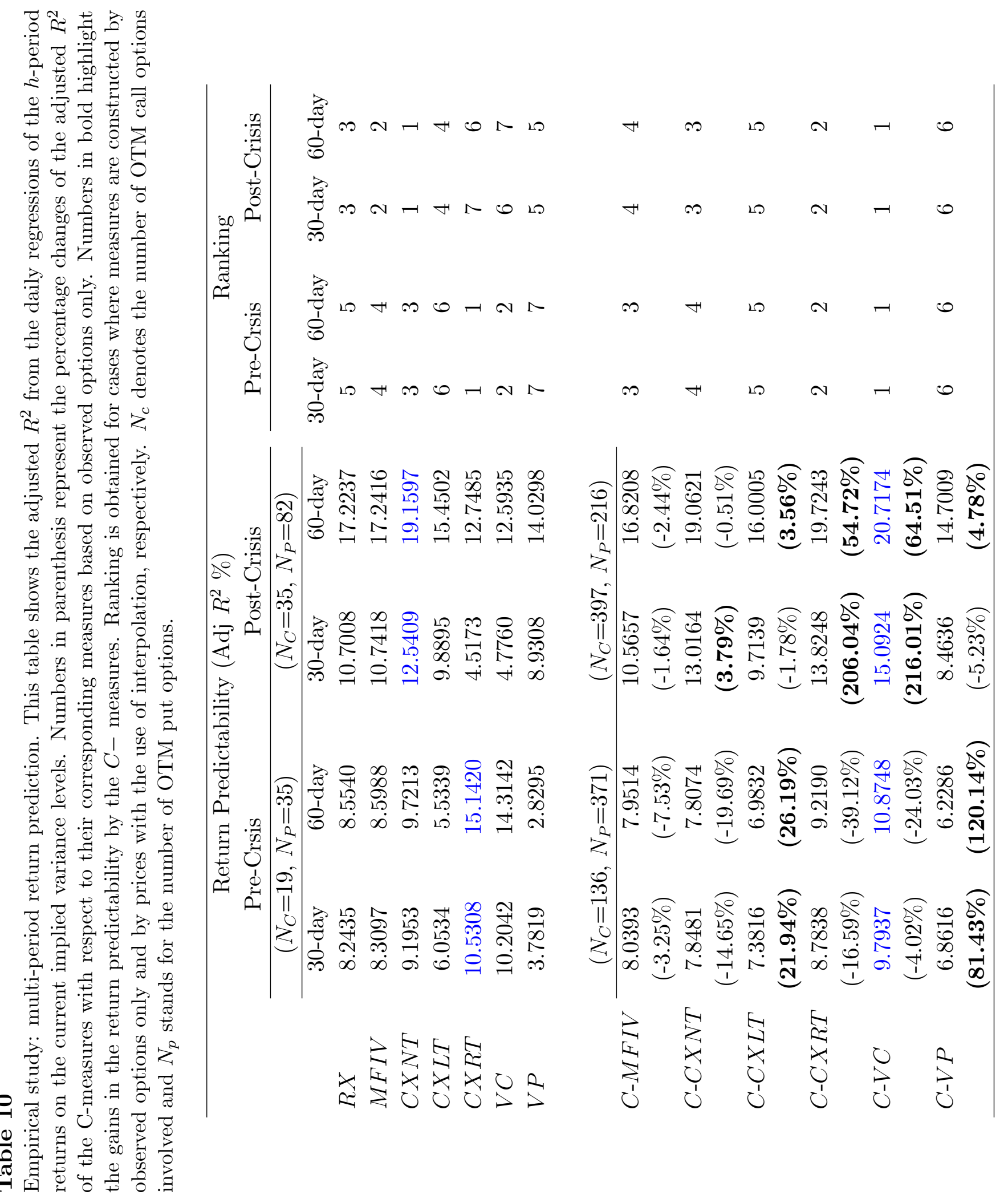



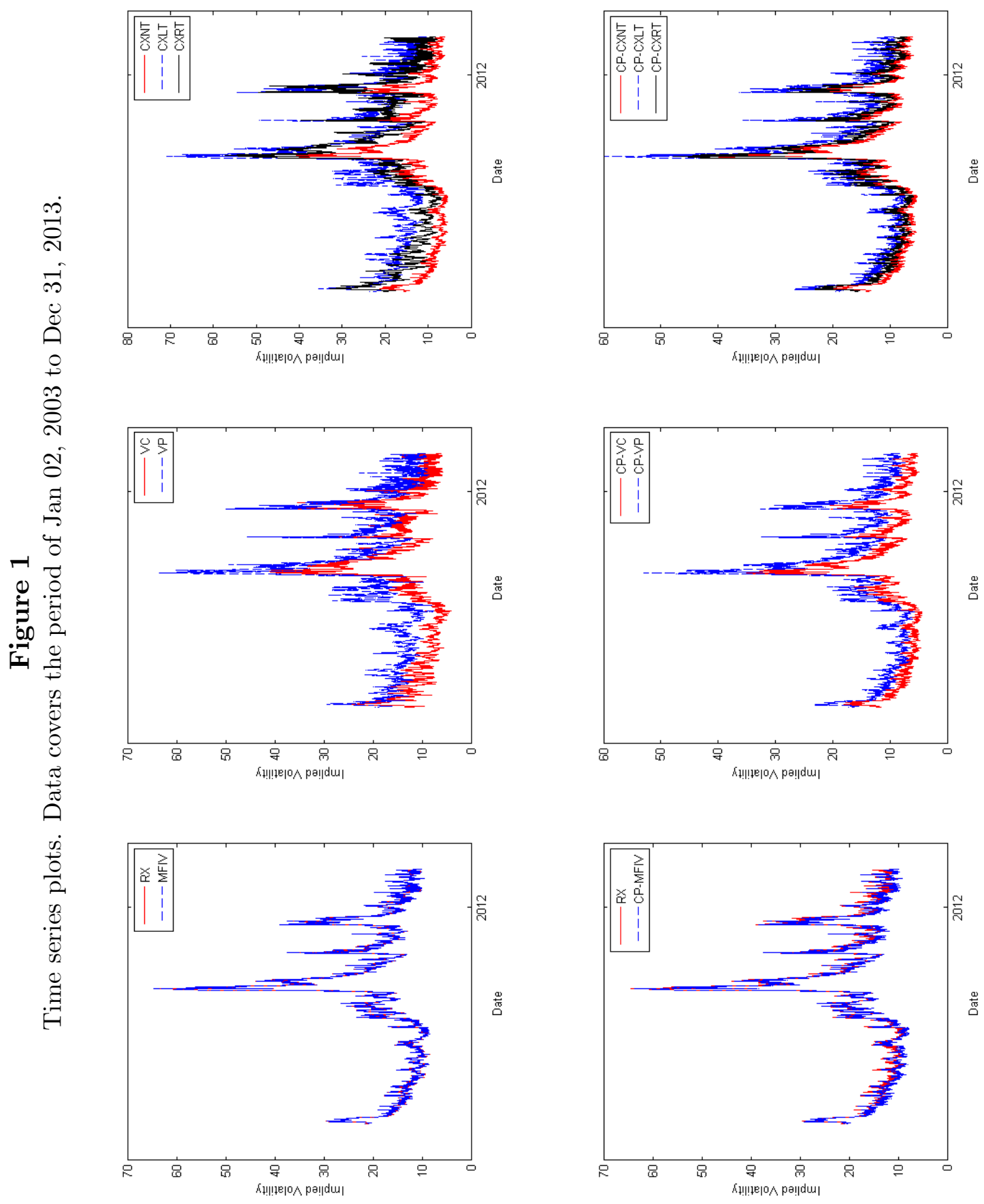